
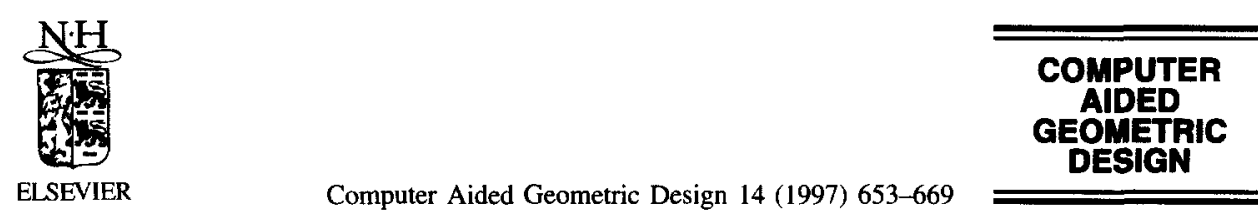

\title{
On convexity of planar curves and its application in CAGD
}

\author{
Chaoyang Liu ${ }^{\mathrm{a}}$, C.R. Traas ${ }^{\mathrm{b}, *}$ \\ ${ }^{a}$ Department of Mathematics, Zhengzhou University, Zhengzhou, 450052, China \\ ${ }^{b}$ Department of Applied Mathematics, University of Twente, Postbus 217, 7500 AE Enschede, \\ The Netherlands \\ Received September 1995; revised June 1996
}

\begin{abstract}
This paper presents a necessary and sufficient condition for global convexity of planar curves and some applications of this condition. In addition, various ideas about the concept of convexity in CAGD are discussed. (c) 1997 Elsevier Science B.V.

Keywords: Parametric curves; Supporting lines; Open or closed curves; Local convexity; Global convexity; Convexity preserving
\end{abstract}

\section{Introduction}

In computer aided geometric design (CAGD), convexity is important as an intuitive geometric concept. In the literature on CAGD this concept is described in diverse ways, or it is used without an explicit definition (Farin, 1993; Wang, 1989; Liu, D., 1980; Goodman, 1989; Shi, 1994).

Definitions on convexity usually are tied with the mathematical disciplines considered: functional analysis, differential and integral geometry, and CAGD. Although the analysis of convexity made in the first three disciplines has some application in CAGD, a further revision of the concept will be helpful for answering some typical questions in CAGD. One point, for example, is that in differential and integral geometry (Berger, 1988; Wu, 1983) and integral geometry (Ren, 1988; Santol, 1988), mainly the convexity of planar, simple, closed curves is investigated. In functional analysis the concept of convex function is based on the notion of 'convex set' ((Hartman and Fang, 1993) and any book on this concept), or only the convexity of single-valued functions is considered.

\footnotetext{
*Corresponding author. E-mail: c.r.traas@math.utwente.nl.
} 
Since the parametric curves in CAGD are possibly open, nonsimple or nonsinglevalued, it is not evident that the above concepts of convexity can be used easily in CAGD. In CAGD, many important results about convexity were obtained, such as the convexity of B-spline curves in (Liang, 1982) and (de Boor, 1988); and the convexity of Bézier curves in (Liu, D., 1980). However, these results are connected with special methods are therefore not applicable to general curves.

In the present article the authors introduce definitions of global and local convexity and they compare with other definitions made by several authors (Section 2). A necessary and sufficient condition is given for global convexity of a general planar curve, whether open or closed, simple or nonsimple (Section 3). Some applications of this condition are given in Section 4.

\section{Convexity for planar curves}

A planar curve is defined as follows.

Definition 2.1. An oriented planar curve is an ordered set in $\mathbb{R}^{2}$, given by

$$
P(t)=[x(t), y(t)], \quad t \in[0,1],
$$

with direction from $t=0$ to $t=1$.

If $P(u)=P(v)$ with $0<u \neq v<1$, then $P(u)$ is a selfintersection point; if $P(t)$ is differentiable at $P(u)$ and $P^{\prime}(u)=0$, then $P(u)$ is an irregular point.

If $P(t)$ has no selfintersection point, then $P(t)$ is simple. If $P(t)$ is differentiable and has no irregular points, then $P(t)$ is said to be regular. Continuous and smooth curves, or simply, $C^{l}$-continuous curves with $l>0$, can be defined in the usual way. Discontinuity of a curve is not excluded.

As shown in Fig. 1, a planar curve may have supporting lines, which are defined as follows.

Definition 2.2. A global supporting line of an oriented curve $P(t)$ at point $P\left(t_{0}\right)$ is an oriented line, $L$, having consistent direction with $P(t)$ in $t_{0}$, and satisfying

(1) $P\left(t_{0}\right)$ is a point of $L$;

(2) The entire curve $P(t), t \in[0,1]$, lies in one closed half-plane with respect to $L$.

A consistent direction on $L$ in $t_{0}$ is the direction obtained by projection of the direction of $P(t)$ in a 'left' or 'right' neighborhood of $t_{0}$, onto $L$, where the choice for left or right is determined by the smallest 'distance' between neighborhood and $L$.

$L$ is a local supporting line if (2) is replaced by

(2') A local neighborhood $P(t), t \in\left[t_{1}, t_{2}\right]$, of $P\left(t_{0}\right)$, lies in one closed half-plane with respect to $L$, where $t_{1}$ and $t_{2}$ satisfy

$$
\begin{aligned}
& 0 \leqslant t_{1}<t_{0}<t_{2} \leqslant 1 \text { or } 0=t_{1}=t_{0}<t_{2} \leqslant 1 \text { or } \\
& 0 \leqslant t_{1}<t_{0}=t_{2}=1 .
\end{aligned}
$$




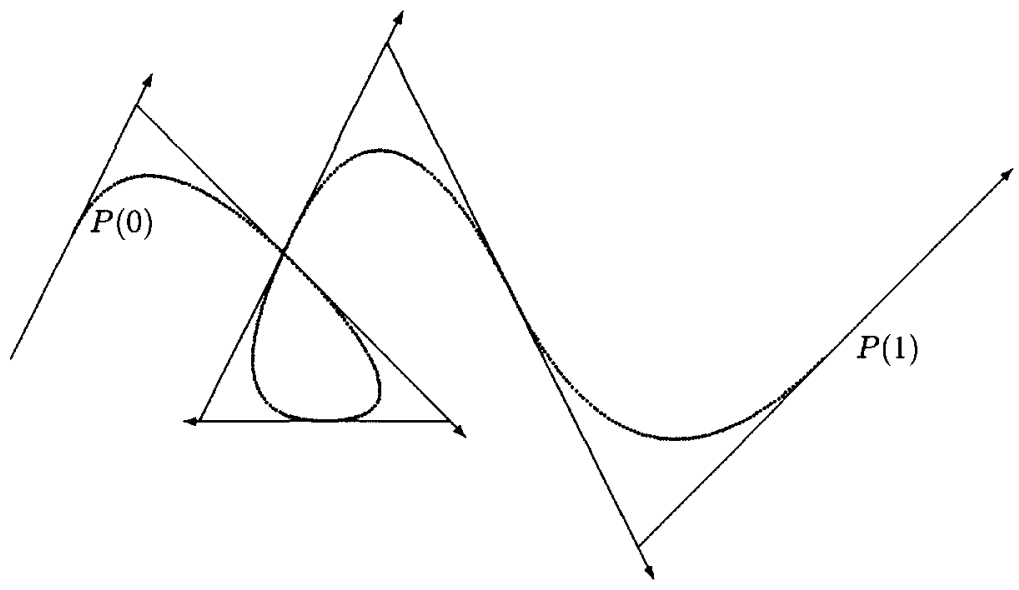

Fig. 1. Curve and supporting lines, nonsupporting line.

Remark 2.3. In contrast to the definition of supporting line in (Ren, 1988), we here insist on the direction of a supporting line. It is easy to show that a tangent line at a point, when it does exist, is the only possible supporting line at this point.

From Definition 2.2, it follows that any global supporting line is a local one. The converse is not true.

If a tangent line exists at a given point and this point is not an inflection point, then it is a local supporting line (see Fig. 1).

Definition 2.4. $P(t), t \in[0,1]$, is a globally convex curve if it satisfies:

(1) There is at least one global supporting line at every point of $P(t)$;

(2) The entire curve lies in the right closed half-plane with the supporting line as its left boundary, as shown in Fig. 2(a).

$P(t), t \in[0,1]$, is a locally convex curve if in (1) the global supporting line is replaced by a local one, and (2) is true only for a related local neighborhood, as shown in Fig. 2(b).

Similarly, a concave curve can be defined as in Definition 2.4 with 'left' and 'right' interchanged.

From Definition 2.2 , it follows that any globally convex curve is locally convex. But the converse is not true.

Remark 2.5. The convexity of a curve depends on its direction. By inverting the direction, a convex curve turns into a concave one. In this way, it is a little odd that a closed convex curve also can be seen as a concave one. But without direction, convex and concave curves can not be identified properly.

In (Farin, 1993) a convex curve is defined as a part of the boundary of a convex set. Let us derive that the boundary of a convex set, if properly oriented, is a globally convex 


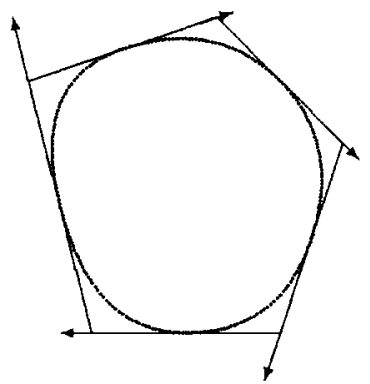

closed smooth curve

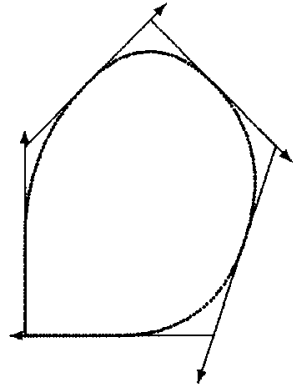

curve with $P(0)=P(1)$

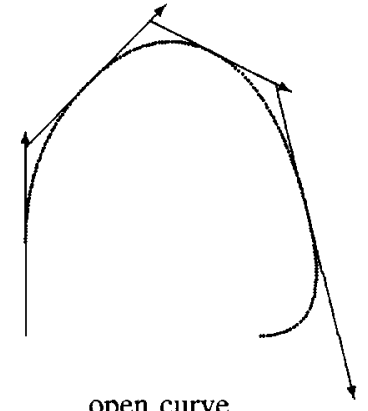

open curve

(a) Globally convex curves

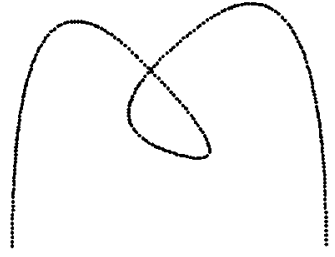

nonsimple curves

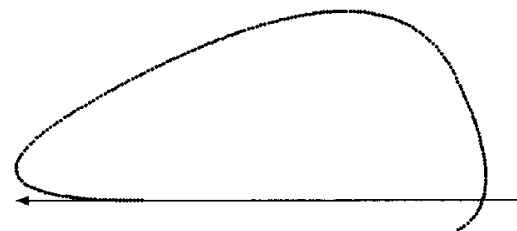

simple curves

(b) Locally convex curves

Fig. 2. Various convex curves.

curve. In (Ren, 1988), supporting lines are defined for boundary points of a convex set. There the whole set, and thus the boundary, lies in one half-plane of any supporting line. That is, a supporting line is a global supporting line of the boundary curve of the set. The Hahn-Banach theorem (see for instance (Ren, 1988)) states that at least one supporting line exists at every boundary point of a convex set. This implies that the boundary curve of a convex set is a global convex curve by Definition 2.4 if the curve is oriented properly. So, the definition in Farin (1993) is included in Definition 2.4. A convex curve is shown in Fig. 3(a). The same curve with different orientation, as shown in Fig. 3(b), is not convex. Anyway, it is not easy to verify the convexity of a concrete curve in CAGD by Farin's definition.

Another widespread definition for a convex curve $P(t)$ is that it is a curve such that any straight line crosses $P(t)$ at most twice (Goodman, 1989). Then a curve which contains a straight line segment should be specially considered. One advantage of this definition is that the convexity property is a direct result of the variation diminishing property, a well-known property of B-spline curves proved by Schoenberg (1953) and of $\beta$-spline curves proved by Goodman (1985). Using this property, it follows with this definition that Bézier and B-spline curves are convex if the corresponding control polygons are convex. This is the typical argument used in CAGD on the convexity of Bézier curves. Since this definition can not distinguish a convex curve from a concave one, the convexity- 


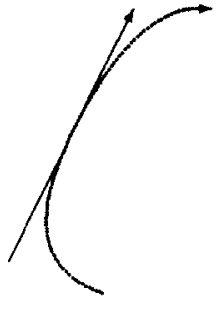

(a) Convex curve
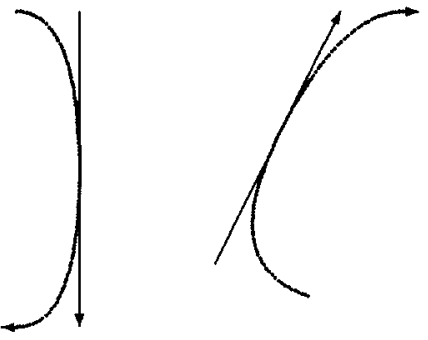

(b) Nonconvex curve

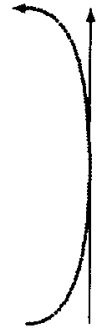

Fig. 3. Convexity of discontinuous curves.

preserving property only ensures that a convex control polygon generates a convex curve. This convex curve might be concave by Definition 2.4. Example 4.2 below presents curves which are concave while the corresponding control polygons are convex. Actually, Bézier and B-spline curves preserve convexity in the sense of Definition 2.4. Liu, C. and Traas (1996) present a geometric proof of the convexity preserving property of nonuniform rational B-spline curves. We remark that the variation diminishing property heavily depends on the existence of a control polygon, while convexity of a curve exists independent of a control polygon.

In (Liu, D., 1980), a convex curve is a locally convex one without any singular point ${ }^{1}$. In general, such a curve is not globally convex, as shown in Fig. 8(a).

We here give some results about planar, regular, simple, closed convex curves as presented in (Wu, 1983) and in other textbooks about differential geometry.

Lemma 2.6. A line segment from $P\left(t_{1}\right)$ to $P\left(t_{2}\right)$ is a part of $P(t)$ if the tangent lines at $P\left(t_{1}\right)$ and $P\left(t_{2}\right)$ coincide.

Lemma 2.7. A simple closed regular $C^{2}$-continuous curve is globally convex if and only if the curvature at every point on the curve is nonpositive.

Remark 2.8. Lemma 2.7 is also true for a $G^{2}$-continuous curve. The reason is that the curvature of a curve is independent of its parameter and that a $G^{2}$-continuous curve is $C^{2}$-continuous with respect to the arc-length parameter. For more details about $G$ - and $C$-continuity, see (de Boor, 1988; Shi, 1994).

In the following sections only planar regular curves are considered, unless stated differently.

\section{A necessary and sufficient condition for convex curves}

In CAGD, there is a result about the local convexity of planar curves.

\footnotetext{
${ }^{1}$ A point $P(t)$ is singular if it is an irregular, or selfintersection point.
} 
Theorem 3.1. $P(t), t \in[0,1]$, is locally convex if and only if

$$
P^{\prime}(t) \times P^{\prime \prime}(t) \leqslant 0, \quad t \in[0,1],
$$

where $P(t)$ is $C^{2}$-continuous, $P^{\prime}(t), P^{\prime \prime}(t)$ are first and second derivatives of $P(t)$, and

$$
P^{\prime}(t) \times P^{\prime \prime}(t)=\left|\begin{array}{cc}
x^{\prime}(t) & x^{\prime \prime}(t) \\
y^{\prime}(t) & y^{\prime \prime}(t)
\end{array}\right|=x^{\prime}(t) y^{\prime \prime}(t)-y^{\prime}(t) x^{\prime \prime}(t) .
$$

A well-known fact is that (3.1) is true if and only if vector $P^{\prime}(t)$ is at the left side of vector $P^{\prime \prime}(t)$ (including that the two vectors are parallel).

From Remark 2.3, the only possible local supporting lines here are tangent lines, therefore convexity is equivalent to the fact that the sufficiently small neighbourhoods always lie at the right sides of the tangent lines. Therefore the proof of this theorem is easy and is omitted.

Wang (1989) defines a convex curve by imposing (3.1). Actually, this definition produces only locally convex curves.

Let us begin with the following lemma.

Lemma 3.2. There exists a globally convex regular curve, which we will call $P_{c}(t)$, $t \in[0,1]$, with given unit tangent vectors $P_{0}^{\prime}, P_{1}^{\prime}$, and curvatures $k_{0} \leqslant 0, k_{1} \leqslant 0$ at the end points $P_{c}(0)$ and $P_{c}(1)$, if

$$
\begin{aligned}
& P_{0}^{\prime} \times\left(P_{c}(1)-P_{c}(0)\right) \leqslant 0 \\
& \quad\left(P_{c}(1)-P_{c}(0)\right) \times P_{1}^{\prime} \leqslant 0 . \\
& P_{c}(t) \text { satisfies } P_{c}(t) \neq P_{c}(0) \text { for } 0<t \leqslant 1 \text { if } P_{c}(0) \neq P_{c}(1) .
\end{aligned}
$$

Proof. Suppose the stated conditions are satisfied.

If the half tangent line at $P_{c}(0)$ and the inverse half tangent line at $P_{c}(1)$ do not intersect, $M_{0}$ and $M_{1}$ are defined as the infinite points of these half lines.

Otherwise, as shown in Fig. 4, the points $M_{0}$ and $M_{1}$ are chosen as follows

$$
P_{0}=P_{c}(0), \quad P_{5}=P_{c}(1) \text {. }
$$

$M$ is the intersection point of the tangent lines at $P_{c}(0)$ and $P_{c}(1)$. Then define $M_{0}=$ $M_{1}=M$.

By (3.3) and (3.4), both $M_{0}$ and $M_{1}$ are at the left of $P_{c}(1)-P_{c}(0)$.

$A$ and $B$ are between $M_{0}$ and $P_{0}, M_{1}$ and $P_{5}$ respectively, and $A B$ is parallel to $P_{0} P_{5}$, $P_{1}, P_{4}$ are between $P_{0}$ and $A, P_{5}$ and $B$ respectively. $P_{2}, P_{3}$ are between $A$ and $B$ such that $A, P_{2}, P_{3}$ and $B$ are ordered points on $A B$.

Since $P_{0} P_{1} P_{2} P_{3} P_{4} P_{5}$ is globally convex, the related Bézier curve is globally convex ${ }^{2}$, too. Denote this curve as $P_{c}(t)$, then $P_{c}(t)$ has given endpoints and tangent lines at the endpoints.

\footnotetext{
${ }^{2}$ For reference, see (Liu, C. and Traas, 1996), where the convexity preserving property of NURBS curves is proved.
} 


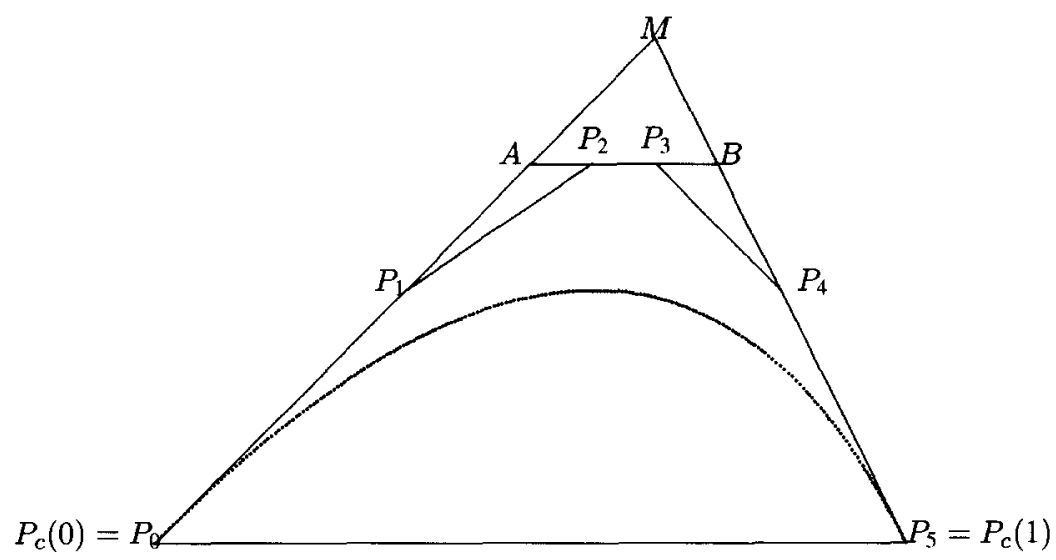

Fig. 4. Show the existence of a convex curve $P_{c}(t)$.

As for curvatures, note that

$$
k(0)=-\frac{4}{5} \frac{\left(P_{2}-P_{1}\right) \times\left(P_{1}-P_{0}\right)}{\left|P_{1}-P_{0}\right|^{3}} \leqslant 0 .
$$

If $k(0)>k_{0}$, move $P_{1}$ towards $P_{0}$. Because

$$
P_{1} \rightarrow P_{0} \text { implies } k(0) \rightarrow-\infty,
$$

$P_{1}$ can be moved until

$$
k(0)=k_{0} \text {. }
$$

If $k(0)<k_{0} \leqslant 0$, move $P_{2}$ towards $A$. Because

$$
P_{2} \rightarrow A \text { implies } k(0) \rightarrow 0,
$$

$P_{2}$ can be moved until $k(0)=k_{0}$. Therefore there exist $P_{1}, P_{2}$ such that $k(0)=k_{0}$ with $P_{0}$ and the tangent line at $P_{0}$ unchanged. In this process, the convexity of the control polygon is preserved, and $P_{3}, P_{4}, P_{5}$, hence $k(1)$ are unchanged.

Similarly, there are $P_{3}, P_{4}$ such that $k(1)=k_{1}$, with the convexity of the control polygon preserved, and $k(0)$ unchanged.

The other properties of $P_{c}(t)$ can be found in (Liu, D., 1980), although we have a different definition of convexity.

The following relationships between chords and tangents exist.

Theorem 3.3. If $P(t)$ is globally convex, then inequalities (3.9)-(3.11) are satisfied.

(1) $P^{\prime}(t) \times P^{\prime \prime}(t) \leqslant 0, \quad t \in[0,1]$

(2) $(P(t)-P(0)) \times P^{\prime}(t) \leqslant 0, \quad t \in[0,1]$,

(3) $P^{\prime}(0) \times(P(t)-P(0)) \leqslant 0, \quad t \in[0,1]$. 
Proof. The global convexity of $P(t)$ implies the local convexity of $P(t)$, hence (1) is true by Theorem 3.1 .

Note that $P^{\prime}(t)$ is the tangent vector, hence the direction of the supporting line at $P(t)$. By Definition 2.4, $P(0)$ is at the right side of the supporting line, therefore,

$$
(P(t)-P(0)) \times P^{\prime}(t) \leqslant 0 .
$$

As for (3), note that $P(t)$ is at the right side of supporting line at $P(0)$.

Actually, (3.10) and (3.11) are also true if $P(0)$ is replaced by any $P\left(t_{0}\right)$ with $0 \leqslant$ $t_{0} \leqslant 1$. For convenience below however, we prefer the above inequalities rather than more general ones.

The propositions below are extensions of Lemma 2.6 .

Proposition 3.4. If $P(t)$ is globally convex and $\left(P\left(t_{0}\right)-P(0)\right) \times P^{\prime}\left(t_{0}\right)=0$ for some $t_{0}, 0 \leqslant t_{0} \leqslant 1$, at least one of the following statements is true.

(1) $P\left(t_{0}\right)=P(0)$;

(2) $P\left(t_{0}\right)=P(1)$

(3) $P(t)$ lies on the line segment $P(0) P\left(t_{0}\right)$ for all $t \in\left[0, t_{0}\right]$;

(4) $P(t)$ lies on the line segment $P\left(t_{0}\right) P(1)$ for all $t \in\left[t_{0}, t_{1}\right]$, where $t_{1}$ satisfies $t_{1}=1$ or $P\left(t_{1}\right)=P(1)$.

Proof. If $P\left(t_{0}\right)=P(0),\left(P\left(t_{0}\right)-P(0)\right) \times P^{\prime}\left(t_{0}\right)=0$ is always true.

Now assume that $P\left(t_{0}\right) \neq P(0)$, then $\left(P\left(t_{0}\right)-P(0)\right) \times P^{\prime}\left(t_{0}\right)=0$ implies that $P^{\prime}\left(t_{0}\right)$ is parallel to the line segment $P\left(t_{0}\right) P(0)$. Therefore $P\left(t_{0}\right) P(0)$ is on the tangent line at $P\left(t_{0}\right)$.

If the direction of the tangent line at $P\left(t_{0}\right)$ is from $P(0)$ to $P\left(t_{0}\right)$, the entire curve lies at the right side of this tangent by Definition 2.4. So the tangent line at $P(0)$ cannot be directed to the left of the tangent line at $P\left(t_{0}\right)$. This implies

$$
P^{\prime}(0) \times\left(P\left(t_{0}\right)-P(0)\right) \geqslant 0 .
$$

In view of (3.11) we have

$$
P^{\prime}(0) \times\left(P\left(t_{0}\right)-P(0)\right)=0 .
$$

Let $s \in\left[0, t_{0}\right]$ such that $P(s)$ has the greatest distance to the line $P(0) P\left(t_{0}\right)$ among all points $P(t), t \in\left[0, t_{0}\right]$. We show that this distance must be zero. The tangent line at $P(s)$ is parallel to $P(0) P\left(t_{0}\right)$, and $P(0)$ lies at the left of this tangent. But in view of global convexity, $P(0)$ should lie at the right side. This implies that $P(s)$ is on the line $P(0) P\left(t_{0}\right)$ and thus (3) is true with $t_{1}=0$. This situation is shown in Fig. 5(a).

On the other hand, the tangent line at $P\left(t_{0}\right)$ can be directed from $P\left(t_{0}\right)$ to $P(0)$. If $t_{0}=1$, then (3) is true, as illustrated in Fig. 5(b).

If $t_{0} \neq 1$, consider a composed closed curve given by $P(t)$ with $t \in\left[0, t_{0}\right]$ and a line segment $P\left(t_{0}\right) P(0)$, as shown in Fig. 5(c). This composed curve is globally convex. The curved part is at the right of $P\left(t_{0}\right) P(0)$, the tangent line at $P\left(t_{0}\right)$. Let $K=\left\{t \in\left[t_{0}, 1\right] \mid P(t)=P(0)\right\}$. If $K$ is empty then take $t_{1}=1$. Otherwise take $t_{1}=\min K$. Then the segment $P(t)$ with $t \in\left(t_{0}, t_{1}\right)$ is at the right side of $P\left(t_{0}\right) P(0)$. 


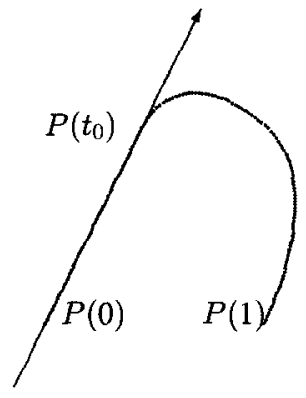

(a) with line segment $P(0) P\left(t_{0}\right)$

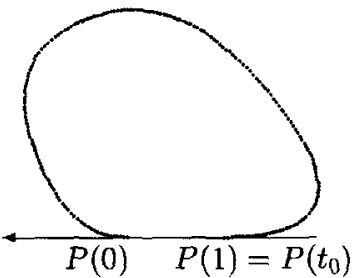

(b) without line segment

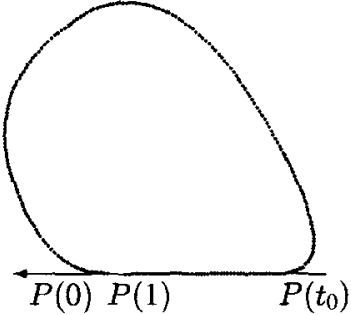

(c) with line segment $P\left(t_{0}\right) P(1)$

Fig. 5. $(P(t)-P(0)) \times P^{\prime}\left(t_{0}\right)=0$ in convex curves.

If this segment is not a part of the line $P\left(t_{0}\right) P(0)$, there is at least one interior point of the composed closed curve such that $P(t)$ with $t \in\left(t_{0}, t_{1}\right)$ passes through this interior point. The tangent line at this interior point has at least one intersection point with the segment $P(t), t \in\left(0, t_{0}\right)$. Then the entire curve can not be at the right side of the tangent line at the interior point, which contradicts the assumption of global convexity of $P(t)$.

Proposition 3.5. If $P(t)$ is globally convex and $P^{\prime}(0) \times\left(P\left(t_{0}\right)-P(0)\right)=0$ for some $t_{0}, 0 \leqslant t_{0} \leqslant 1$, at least one of the following statements is true.

(1) $P\left(t_{0}\right)=P(0)$;

(2) $P\left(t_{0}\right)=P(1)$;

(3) $P(t)$ lies on the line segment $P(0) P\left(t_{0}\right)$ for all $t \in\left[0, t_{0}\right]$;

(4) $P(t)$ lies on the line segment $P\left(t_{0}\right) P(1)$ for all $t \in\left[t_{0}, t_{1}\right]$, where $t_{1}$ satisfies $t_{1}=1$ or $P\left(t_{1}\right)=P(1)$.

The proof of this proposition is similar to that of Proposition 3.4 and is omitted.

Lemma 3.6. If $P(t)$ satisfies inequalities (3.9)-(3.11), and

$$
P(t) \neq P(0) \text { for } t \in(0,1),
$$

then $P(t)$ is simple. 
Proof. By (3.9), $P(t)$ is locally convex.

Suppose that the conclusion is not true, then $P(t)$ has at least one selfintersection point, i.e., there are $t_{1}, t_{2}$ such that

$$
P\left(t_{1}\right)=P\left(t_{2}\right) \neq P(0), \quad 0<t_{1}<t_{2}<1 .
$$

Consider the segment curve $\Gamma: P(t)$ with $0<t_{1} \leqslant t \leqslant t_{2}<1$. $\Gamma$ is a closed curve. If $\Gamma$ and $P(0)$ are on a common line then it easy to show that the farthest point of $\Gamma$ away from $P(0)$ would be an irregular point, which contradicts the assumption of regularity of $P(t)$.

Otherwise, there exists at least one line at $P(0)$ such that $\Gamma$ extends to both sides of this line. Chosen a point $A \neq P(0)$ on this line, we obtain a oriented line $P(0) A$, with direction from $P(0)$ to $A$. Because $\Gamma$ is a planar close curve, there is at least one intersection point of $\Gamma$ with $P(0) A$, such that $\Gamma$ crosses $P(0) A$ from the right to the left. Assume that the intersection point is $P\left(t_{0}\right), t_{0} \in\left[t_{1}, t_{2}\right]$, then

$$
(A-P(0)) \times P^{\prime}\left(t_{0}\right) \geqslant 0 \text {. }
$$

By (3.11), the whole curve is located at the right of $P^{\prime}(0)$, i.e., the tangent line at $P(0)$. This implies that both $P\left(t_{0}\right)$ and $A$ are at the same side of $P^{\prime}(0)$, therefore there is an $\alpha \geqslant 0$, such that

$$
P\left(t_{0}\right)-P(0)=\alpha(A-P(0))
$$

and

$$
\left(P\left(t_{0}\right)-P(0)\right) \times P^{\prime}\left(t_{0}\right)=\alpha(A-P(0)) \times P^{\prime}\left(t_{0}\right) \geqslant 0 .
$$

Hence in view of $(3.10)$,

$$
\left(P\left(t_{0}\right)-P(0)\right) \times P^{\prime}\left(t_{0}\right)=0 .
$$

Note that $0<t_{1}<1$,

$$
P\left(t_{0}\right)-P(0) \neq 0 \text {. }
$$

This implies that the common lines $P\left(t_{0}\right)-P(0)$ and $P(0) A$ are the tangent line at $P\left(t_{0}\right)$.

From (3.9), $P(t)$ is locally convex. Hence, as a part of $P(t), \Gamma$ cannot cross $P(0) A$ at $P\left(t_{0}\right)$, except that there is a common segment of $P(0) A$ and of $\Gamma$ around $P\left(t_{0}\right)$.

Because $\Gamma$ is at different sides of $P(0) A$, a part of $\Gamma$ consists of a line segment and two curved segments at two endpoints of the line segment, at different sides of the line segment. This part of $\Gamma$ cannot be locally convex at one of the two endpoints. This result contradicts the local convexity of $P(t)$. Hence, a selfintersection point is impossible.

Lemma 3.7. If $P(t)$ is a closed curve satisfying inequalities (3.9)-(3.11) and (3.13), then $P(t)$ is globally convex.

This is a direct result of Lemmas 3.6 and 2.7.

Lemma 3.8. An open curve $P(t)$ is globally convex if (3.9)-(3.11) and (3.13) are satisfied. 
Proof. From (3.10) and (3.11),

$$
P^{\prime}(0) \times(P(1)-P(0)) \leqslant 0, \quad(P(1)-P(0)) \times P^{\prime}(1) \leqslant 0,
$$

i.e.,

$$
P^{\prime}(1) \times(P(0)-P(1)) \leqslant 0, \quad(P(0)-P(1)) \times P^{\prime}(0) \leqslant 0 .
$$

This implies a new convex curve $P_{c}(s), s \in[0,1]$, satisfying the conditions of $P(t)$ at $P(1)$ and $P(0)$ by Lemma 3.2. Since $P(t)$ is open, $P(0) \neq P(1)$, also by Lemma 3.2,

$$
P_{c}(s) \neq P_{c}(1)=P(0) \text { for } 0<s \leqslant 1 \text {. }
$$

On the basis of the comments following Theorem 3.3, $P_{c}(s)$ satisfies (3.9)-(3.11) with $P_{c}(1)=P(0)$.

Now the curves $P_{c}(s)$ and $P(t)$ together form a closed, $G^{2}$-continuous curve satisfying the conditions in Lemma 3.7 , hence it is a globally convex curve.

The global convexity of $P(t)$ follows from the fact that it is a part of a closed globally convex curve.

Combining Theorem 3.3 with the Lemmas 3.7 and 3.8, the main result can be stated as follows.

Theorem 3.9. A curve $P(t)$ satisfying (3.13) is globally convex if and only if (3.9), (3.10), (3.11) are satisfied, whether the curve is open or closed.

Remark 3.10. Inequality (3.13) is implied by either of the following inequalities.

$$
P^{\prime}(0) \times(P(t)-P(0)) \neq 0, \quad t \in(0,1) ;
$$

or

$$
(P(t)-P(0)) \times P^{\prime}(t) \neq 0, \quad t \in(0,1) .
$$

(3.20) or (3.21) can be easily verified using (3.10) or (3.11). In many cases, both (3.20) and (3.21) are true.

The following Corollaries 3.11-3.12 are more convenient in applications than Theorem 3.9 .

Corollary 3.11. $P(t)$ is globally convex if (3.9), (3.10) and

$$
P^{\prime}(0) \times(P(t)-P(0))<0, \quad t \in(0,1) ;
$$

are satisfied.

Evidently, (3.22) implies (3.20), hence, (3.13). It also implies (3.11) by a continuity argument. The conclusion follows from Theorem 3.9.

A similar conclusion is 


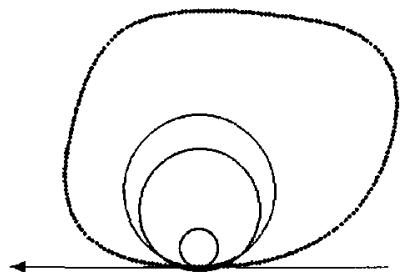

Fig. 6. A nonconvex curve without (3.13).

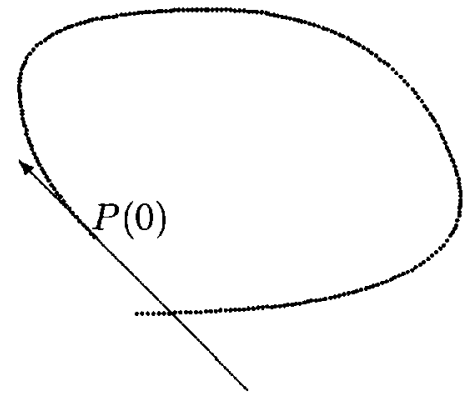

Fig. 7. A simple, nonconvex curve without (3.11).

Corollary 3.12. $P(t)$ is globally convex if (3.9), (3.11) and

$$
(P(t)-P(0)) \times P^{\prime}(t)<0, \quad t \in(0,1) .
$$

are satisfied.

Remark 3.13. Eq. (3.13) is necessary for Theorem 3.9. If (3.13) is not true, the conclusion may be wrong. A composed curve, produced by many different closed convex curves with point $P(0)=P(1)$ and common tangent line $P^{\prime}(0)=P^{\prime}(1)$ at the common point, satisfies (3.9), (3.10) and (3.11), but is not a globally convex curve, as shown in Fig. 6, unless all these closed curves coincide. This is the only kind of globally convex curves which is not implied by Theorem 3.9 .

Remark 3.14. In part of the literature, such as (Liu, D., 1980), an open curve is defined as convex if it has nonpositive curvatures, without any singular points. This curve is only simple and locally convex, but in general not globally convex (Certainly, it is globally convex from Lemma 2.7 , if it is closed).

An example is shown in Fig. 7. For this simple curve, (3.13), (3.9) and (3.10) are true, but (3.11) is not true, because the curve lies at different sides of $P^{\prime}(0)$.

Remark 3.15. Nonconvex curves with (3.13), (3.9) and (3.11), but without (3.10), are shown in Fig. 8. 


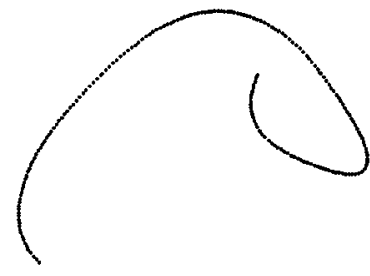

(a) Simple curve

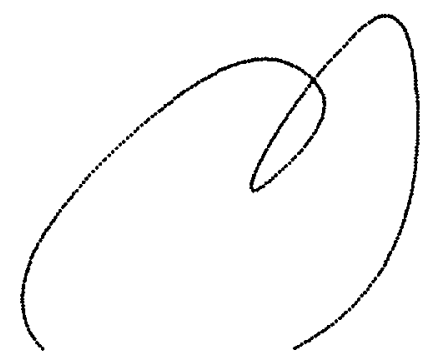

(b) Nonsimple curve

Fig. 8. Nonconvex curves without (3.10).

\section{Examples}

Theorem 3.3 can be easily used to identify the convexity of concrete parametric curves. Here are two examples.

Example 4.1. Take $P_{0}=(\alpha, 0), P_{1}=(-1,1), P_{2}=(1,1), P_{3}=(-\alpha, 0)$, then the polygon given by these four points is always locally convex; but globally convex only if $\alpha \leqslant 0$. Also it is open if $\alpha<0$; closed if $\alpha=0$ and selfintersecting if $\alpha>0$. Now we analyze the cubic Bézier and cubic B-spline curves produced by this control polygon.

The cubic Bézier curve is

$$
P(t)=\left(\begin{array}{l}
(-6-2 \alpha) t^{3}+(9+3 \alpha) t^{2}-(3+3 \alpha) t+\alpha \\
-3 t^{2}+3 t
\end{array}\right),
$$

therefore,

$$
P^{\prime}(t) \times P^{\prime \prime}(t)=-36(1-(3+\alpha) t(1-t)) \leqslant 0
$$

if and only if

$$
\alpha \leqslant 1 \text {; }
$$

$$
(P(t)-P(0)) \times P^{\prime}(t)=-18\left((1+\alpha)(1-t)^{2}-\alpha\right) \leqslant 0
$$

if and only if

$$
\begin{aligned}
& \alpha \leqslant 0 \\
& P^{\prime}(0) \times(P(t)-P(0))=-6 t^{2}(3-(3+\alpha) t) \leqslant 0
\end{aligned}
$$

if and only if

$$
\alpha \leqslant 0 \text {. }
$$

Hence, this cubic Bézier curve is locally convex only if $\alpha \leqslant 1$. Therefore the local convexity of a control polygon does not imply the local convexity of its Bézier curve. It is globally convex if $\alpha \leqslant 0$, i.e., if the control polygon is globally convex. 
If the $P_{i}, i=0, \ldots, 3$, are considered as 4 consecutive control points out of a set of control points associated with an uniform knot-sequence $\{\ldots,-1,0,1, \ldots\}$, then we find the corresponding cubic polynomial spline segment:

$$
P(t)=\frac{1}{6}\left(\begin{array}{l}
(-6-2 \alpha) t^{3}+(9+3 \alpha) t^{2}+(3-3 \alpha) t+\alpha-3 \\
-3 t^{2}+3 t+5
\end{array}\right)
$$

For this segment applies:

$$
P^{\prime}(t) \times P^{\prime \prime}(t)=-2+(3+\alpha) t(1-t) \leqslant 0
$$

if and only if

$$
\alpha \leqslant 5
$$

$$
(P(t)-P(0)) \times P^{\prime}(t)=-\frac{1}{6} t^{2}(6-(3+\alpha) t(2-t)) \leqslant 0
$$

if and only if

$$
\begin{aligned}
& \alpha \leqslant 3 ; \\
& P^{\prime}(0) \times(P(t)-P(0))=-\frac{1}{6} t^{2}(6-(3+\alpha) t) \leqslant 0
\end{aligned}
$$

if and only if

$$
\alpha \leqslant 3 \text {. }
$$

Hence, this cubic B-spline curve is locally convex only if $\alpha \leqslant 5$. Therefore the local convexity of the control polygon does not imply the local convexity of its B-spline curve. It is globally convex if $\alpha \leqslant 3$. This B-spline curve can be seen as a Bézier curve with a control polygon given by

$$
((\alpha-3) / 6,5 / 6),(-1 / 3,1),(1 / 3,1),((3-\alpha) / 6,5 / 6) \text {. }
$$

In case of $\alpha \leqslant 3$, this polygon is globally convex.

This example shows that it is helpful to have a proper concept of convexity whenever we want to use the convexity preserving property of Bézier and B-spline curves.

Example 4.2. With three control points $\left\{P_{i}: i=0,1,2\right\}$ and quadratic polynomial blending functions

$$
g_{i}(t)=g_{i 0}+g_{i 1} t+g_{i 2} t^{2}, \quad i=0,1,2,
$$

a quadratic polynomial parametric curve can be defined as

$$
P(t)=\sum_{i=0}^{2} P_{i} g_{i}(t)
$$

If

$$
\sum_{i=0}^{2} g_{i}(t)=1
$$


$P(t)$ is unchanged under shift and rotation. Therefore assume, without loss of generality, that

$$
P_{0}=(0,0) \text {. }
$$

In this case,

$$
P(t)=P_{1} g_{1}(t)+P_{2} g_{2}(t) .
$$

This polygon is always convex, and the curve is a parabolic curve, thus also convex. Theorem 3.3 confirms this fact in an exact way.

By (4.5),

$$
\left\{\begin{array}{c}
P^{\prime}(t) \times P^{\prime \prime}(t)=\left|\begin{array}{ll}
g_{1}^{\prime}(t) & g_{1}^{\prime}(t) \\
g_{2}^{\prime}(t) & g_{2}^{\prime \prime}(t)
\end{array}\right|=\left|\begin{array}{ll}
g_{11} & g_{12} \\
g_{21} & g_{22}
\end{array}\right| t P_{1} \times P_{2}, \\
P^{\prime}(0) \times(P(t)-P(0))=\left|\begin{array}{ll}
g_{1}^{\prime}(0) & g_{1}(t)-g_{1}(0) \\
g_{2}^{\prime}(0) & g_{2}(t)-g_{2}(0)
\end{array}\right|=\left|\begin{array}{ll}
g_{11} & g_{12} \\
g_{21} & g_{22}
\end{array}\right| t^{2} P_{1} \times P_{2}, \\
(P(t)-P(0)) \times P^{\prime}(t)=\left|\begin{array}{ll}
g_{1}(t)-g_{1}(0) & g_{1}^{\prime}(t) \\
g_{2}(t)-g_{2}(0) & g_{2}^{\prime}(t)
\end{array}\right|=\left|\begin{array}{ll}
g_{11} & g_{12} \\
g_{21} & g_{22}
\end{array}\right| t^{2} P_{1} \times P_{2} .
\end{array}\right.
$$

Note that the control polygon $P_{0} P_{1} P_{2} P_{0}$ is convex if $P_{1} \times P_{2} \leqslant 0$ and concave if $P_{1} \times P_{2} \geqslant 0$. Therefore $P(t)$ preserves the global convexity or concavity of the control polygon if and only if $P^{\prime}(t) \times P^{\prime \prime}(t), P^{\prime}(0) \times(P(t)-P(0))$ and $(P(t)-P(0)) \times P^{\prime}(t)$ have the sign of $P_{1} \times P_{2}$. From (4.6) follows that this is equivalent to

$$
\left|\begin{array}{ll}
g_{11} & g_{12} \\
g_{21} & g_{22}
\end{array}\right| \geqslant 0
$$

which is independent of the parameter $t$.

Specifically, if

$$
\left|\begin{array}{ll}
g_{11} & g_{12} \\
g_{21} & g_{22}
\end{array}\right|=0
$$

$P(t)$ is a line segment.

One thing more is that in case of

$$
\left|\begin{array}{ll}
g_{11} & g_{12} \\
g_{21} & g_{22}
\end{array}\right| \leqslant 0,
$$

$P(t)$ is concave if $P_{0} P_{1} P_{2} P_{0}$ is convex and convex if $P_{0} P_{1} P_{2} P_{0}$ is concave.

A concrete example of this kind is

$$
g_{0}(t)=1-\frac{1}{4} t-\frac{1}{4} t^{2}, \quad g_{1}(t)=\frac{1}{4} t^{2}, \quad g_{2}(t)=\frac{1}{4} t .
$$


Then (4.3) is satisfied and the $g_{i}(t), t \in[0,1], i=0,1,2$, are nonnegative, hence $P(t)$ produced by these blending functions has the convex hull property. But

$$
\left|\begin{array}{ll}
g_{11} & g_{12} \\
g_{21} & g_{22}
\end{array}\right|=\left|\begin{array}{cc}
0 & \frac{1}{4} \\
\frac{1}{4} & 0
\end{array}\right|=-\frac{1}{16}<0 .
$$

Here $P(t)$ does not preserve the convexity of its control polygon.

\section{Conclusion}

Compared with the conditions in Definition 2.4, inequalities (3.9), (3.10) and (3.11) are quite simple. These inequalities are elegant and similar in form. They are widely applicable in CAGD, as shown in Section 4. Also for closed curves, Theorem 3.9 has its own advantage. A restriction of Lemma 2.7 is that the curve should be simple. With Lemma 3.6, this condition is implied by the inequalities. Therefore when using Theorem 3.9 to a concrete curve it is not necessary to check whether there is a selfintersection point or not.

\section{Acknowledgments}

The authors thank the referees for the careful reading of the initial manuscript and for a number of suggestions and comments.

\section{References}

Berger, B. (1988), Differential Geometry: Manifolds, Curves and Surfaces, Springer-Verlag.

de Boor, C. (1988), A Practical Guide to Splines, Springer-Verlag.

Farin, G. (1993), Curves and Surfaces for Computer Aided Geometric Design: A Practical Guide, Academic Press.

Goodman, T.N.T. (1985), Properties of beta-splines, J. Approx. Theory 44, 132-153.

Goodman, T.N.T. (1989), Shape preserving representations, in: Lyche, T. and Schumaker, L.L., eds., Mathematical Methods in CAGD, Academic Press, Boston 1989, 333-357.

Hartman, E., and Fang, Y.Y. (1993), On the convexity of functional splines, Computer Aided Geometric Design 10, 127-142.

Liang, Y. (1982), Theory of B-spline geometric curves and surfaces and their properties of preserving convexity and shape, J. of Zhejiang University (in Chinese).

Liu, D. (1982), A theorem on the convexity of planar Bézier curves, Chinese Ann. of Math. 3, 45-55 (in Chinese).

Liu, C. and Traas, C.R. (1996), A geometric proof for the convexity preserving property of NURBS curves, Submitted.

Ren, D. (1988), Introduction to Integral Geometry, Shanghai Science and Technology Publishing House.

Santol, L.A. (1988), Integral Geometry and Geometric Probability. 
Schoenberg, I. (1953), On variation diminishing approximation methods, in: Langer, R.E., ed., On Numerical Approximation, Univ. of Wisconsin Press, 249-274.

Shi, F. (1994), CAGD and NURBS, Beijing Aerospace University, Publishing House of China (in Chinese).

Sun, J. (1978), Spline Functions and Computational Geometry, Academic Press of China (in Chinese).

Wang, X. (1989), Spline Functions and Applications, West-North Technology University Publishing House of China (in Chinese).

Wu, D. (1983), Textbook of Differential Geometry, Renmin Education Publishing House of China (in Chinese). 